\title{
Compact LWIR polarimeter for cirrus ice properties
}

Kira A. Hart, Russell A. Chipman, Dong L. Wu

Kira A. Hart, Russell A. Chipman, Dong L. Wu, "Compact LWIR polarimeter for cirrus ice properties," Proc. SPIE 10655, Polarization: Measurement, Analysis, and Remote Sensing XIII, 106550V (14 May 2018); doi: 10.1117/12.2305106

Event: SPIE Commercial + Scientific Sensing and Imaging, 2018, Orlando, Florida, United States 


\title{
Compact LWIR polarimeter for cirrus ice properties
}

\author{
Kira A. Hart ${ }^{\mathrm{a}}$, Russell A. Chipman ${ }^{\mathrm{b}}$, and Dong L. $\mathrm{Wu}^{\mathrm{c}}$ \\ a,b University of Arizona, 1630 E University Blvd,Tucson, USA \\ ${ }^{\mathrm{c} N A S A}$ Goddard Space Flight Center, 8800 Greenbelt Rd, Greenbelt, USA
}

\begin{abstract}
A miniaturized long-wave InfraRed (LWIR) spectro-polarimeter is being developed as a prototype for the Compact Submm-Wave and LWIR Polarimeters (SWIRP) project. The polarimeter in development is a compact $(20 \times 20 \times 40 \mathrm{~cm})$ conical-scan instrument to measure the polarimetric radiation from ice cloud scattering at mmsubmm $(220$ and $680 \mathrm{GHz})$ and IR $(8.6,11$, and $12 \mathrm{~m})$ bands. The LWIR polarimeter will provide a series of polarization measurements across the 8.5 - 12.5 micron band, measuring the full set of linear Stokes parameters (I, Q, U) as a function of wavelength. The spectro-polarimeter uses a combination of birefringent crystals, a Wollaston prism, a diffraction grating, and an uncooled microbolometer array to measure both the degree and angle of linear polarization across the spectral bandwidth by modulating the polarization flux in wavelength with a high order retarder.
\end{abstract}

Keywords: channeled spectro-polarimeter, LWIR, ice clouds, remote sensing, Wollaston prism

\section{INTRODUCTION}

Clouds remain a major source of uncertainty in climate models. Lack of accurate measurements of cloud ice and its microphysical properties has led to large uncertainty about global clouds and their processes within the atmosphere. ${ }^{1}$ NASA's Aerosol, Cloud and Ecosystems (ACE), an Earth Science Decadal Survey (DS) mission, recommended an advanced science payload with submm-wave and longwave infrared (LWIR) radiometers for such cloud ice measurements. ${ }^{2}$ In a recent community white paper, Cloud and Precipitation Process Measurements $(\mathrm{CaPPM})$, dynamics and microphysical properties are identified as the key links between the cloud-precipitation processes and need more accurate measurements. The importance of ice cloud observations from submm-wave remote sensing is reaffirmed in the 2017 NRC Decadal Survey [NRC, 2018], ${ }^{3}$ and is recognized by the international community. ${ }^{1}$ The cloud-induced radiances at $220,680 \mathrm{GHz}$ and $12 \mu \mathrm{m}$ bands provide the wide dynamic range in sensitivity needed for measuring the targeted ice particles. Polarimetric measurements at the 8.6, 11, and $12 \mu \mathrm{m}$ bands determine bulk particle shape, which is characterized by the aspect ratio from $\mathrm{H}-$ and Vpolarization differences and can be measured to $5 \%$ or better. The InfraRed Channeled Spectro-Polarimeter (IRCSP) presented here performs polarimetric measurements over these targeted bands.

The construction of the LWIR polarimeter relies on the miniaturization of an IRCSP, which performs a spectrally-dependent polarization modulation to produce a cosinusoidal intensity pattern at the detector whose intensity is proportional to degree of linear polarization (DoLP). ${ }^{4}$ The angle and amplitude of the intensity patterns produced by the ICRSP are used to retrieve the angle of linear polarization (AoLP) and degree of linear polarization with high resolution in the LWIR. ${ }^{5}$ Channeled-polarimeters have been shown to be effective in producing instantaneous hyperspectral polarimetric data without sacrificing spatial resolution and have many remote sensing applications. ${ }^{4,6}$ Iannarilli and Shaw have previously demonstrated a LWIR Polarimetric Hyperspectral Imager capable measuring the DoLP and AoLP of light scattered from ocean surfaces, measuring local surface orientation. ${ }^{5}$ Channeled Spectro-Polarimeters have also been applied to sense ground mines and atmospheric aerosols from space. ${ }^{7,8}$

The SWIRP parameters outline a ICRSP of dimensions $2 \times 2 \times 10 \mathrm{~cm}$ with a mass of $0.5 \mathrm{~kg}$. ICRSP power consumption should be $\leq 0.5 \mathrm{~W}$, with a data output of no more than $50 \mathrm{kbps}$. To achieve this compact and efficient specification, the ICRSP is constructed using a combination of birefringent crystals serving as multi-order waveplates, a Wollaston prism, and a diffraction grating. An uncooled microbolometer is utilized to efficiently

Further author information: (Send correspondence to khart@optics.arizona.edu)

Polarization: Measurement, Analysis, and Remote Sensing XIII, edited by David B. Chenault, Dennis H. Goldstein, Proc. of SPIE Vol. 10655, 106550V · ( 2018 SPIE · CCC code:

0277-786X/18/\$18 $\cdot$ doi: $10.1117 / 12.2305106$

Proc. of SPIE Vol. 10655 106550V-1 
record all of the necessary measurements over just 4 x 40 pixels. The project specifications aim to achieve $0.5 \%$ DoLP accuracy over 90\% of the spectral band and advance the design from TRL 3 to TRL 5. To achieve the desired performance metrics, careful design considerations must be made to account for the radiometric and calibration challenges in the LWIR.

\section{METHODS}

\subsection{Channeled Spectro-Polarimeter Concept}

The channeled spectro-polarimeter is comprised of a series of polarization modulation components followed by a diffraction grating and imaging lens, which image a polarization modulated spectrum at the focal plane. The polarization modulation is performed by a quarter wave retarder (QWR) with a fast axis at $45^{\circ}$ followed by a CdSe crystal high order retarder (HOR). ${ }^{6}$ This combination acts as a wavelength-dependent circular retarder, rotating the linear polarization by different angles as a function of wavelength. Following the HOR is a Wollaston prism also made of $\mathrm{CdSe}$, which separates the $0^{\circ}$ and $90^{\circ}$ polarization states by an angle

$$
\theta_{p s} \approx 2\left(n_{e}-n_{o}\right) \tan \Omega
$$

where $n_{e}$ and $n_{o}$ are the extraordinary and ordinary refractive indicies of the CdSe. The Wollaston prism (WP) is comprised of two prisms cut at an angle $\Omega \sim 10^{\circ}$ whose perpendicular optical axes result in two perpendicular output polarization states separated by $\theta_{p s}$. The measurement of both beams' output by the WP preserves both modulated channels, enabling the distinction between wavelength dependent transmission and polarization effects.

Unpolarized light has its polarization state unchanged by the QWR and HOR, so one half of the flux exits the WP in each outgoing polarization beam for all wavelengths. When linearly polarized light is incident, the output polarization from the QWR and HOR is linear but rotates as a function of the wavelength, as shown in Figure $1 .^{6}$ Then, the WP modulates the transmitted intensity as a function of wavelength with a modulation period of 1 micron with opposite polarities in $0^{\circ}$ and $90^{\circ}$ channels (Equations 3,4 ). The modulation period corresponds to a spectral retardance variation of one wave per micron, which prescribes the length of the HOR, since the specified resolution of the polarization measurement is a 1- $\mu \mathrm{m}$ bandpass centered on $11 \mu \mathrm{m}$. The amount of modulation is proportional to the degree of linear polarization, and the phase of the modulation describes the angle of the linear polarization. Thus in this design, the DoLP and AoLP are measured with $1 / 4-\mu m$ resolution, as rapid variation with wavelength is not expected. Finally, the diffraction grating and imaging lens form a spectrum at the focal planes 40x2 array for each polarization channel, 40 for the spectral sampling and 2 for spatial sampling. The amplitude and phase of the spectral modulation in each 1- $\mu m$ band provides the polarization measurement. The system's idealized Mueller matrix model for the $0^{\circ}$ polarization state is described as

$$
\frac{1}{2}\left[\begin{array}{cccc}
1 & 0 & 1 & 0 \\
0 & 0 & 0 & 0 \\
1 & 0 & 1 & 0 \\
0 & 0 & 0 & 0
\end{array}\right]\left[\begin{array}{cccc}
1 & 0 & 0 & 0 \\
0 & 1 & 0 & 0 \\
0 & 0 & \cos \delta(\lambda) & \sin \delta(\lambda) \\
0 & 0 & -\sin \delta(\lambda) & \cos \delta(\lambda)
\end{array}\right]\left[\begin{array}{cccc}
1 & 0 & 0 & 0 \\
0 & 0 & 0 & -1 \\
0 & 0 & 1 & 0 \\
0 & 1 & 0 & 0
\end{array}\right]\left[\begin{array}{c}
1 \\
D \cos (2 \theta) \\
D \sin (2 \theta) \\
0
\end{array}\right]=\frac{1}{2}\left[\begin{array}{c}
1+D \sin (\delta(\lambda)-2 \theta) \\
0 \\
1+D \cos (\delta(\lambda)-2 \theta) \\
0
\end{array}\right]
$$

where D is the DoLP, $\theta$ is the AoLP, and $\delta(\lambda)$ is the wavelength dependent retardance spectrum of the HOR. The retardance spectrum is designed such that $\delta_{8.5-12.5}=\delta(8.5)-\delta(12.5)=4$ waves (Figure 5). The flux variation at the detector is

$$
\begin{aligned}
& \frac{1}{2}(1+D \sin (\delta(\lambda)-2 \theta)) \\
& \frac{1}{2}(1-D \sin (\delta(\lambda)-2 \theta))
\end{aligned}
$$

for the $0^{\circ}$ (Equation 3) and $90^{\circ}$ (Equation 4) outputs. The total transmission is calculated from the sum of both the $0^{\circ}$ and $90^{\circ}$ polarization intensity patterns at the detector, scaled by the transmission of the optics and efficiency of the diffraction grating. All wavelength dependent system transmission losses are measured and included in the polarimetric data reduction model. The total intensity spectrum is then used to distinguish transmission signatures from the source spectrum and absorption in the atmosphere from the polarization signatures, discussed at length in Section 3.1. 


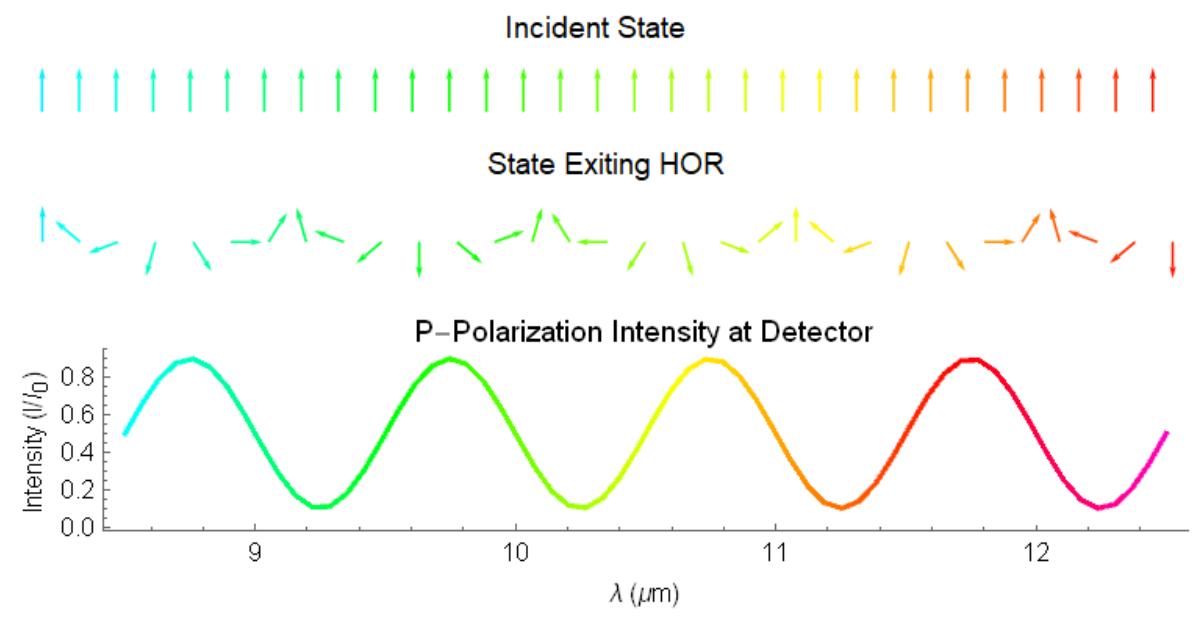

Figure 1. The operation of IRCSP is outlined for one output channel of the Wollaston prism for an incident state of $90^{\circ}$ linearly polarized light (top). Wavelength is encoded as color (blue for the shortest wavelengths, red for the longest). (Middle) The state after transiting the QWR and HOR has the same amplitude but the orientation rotates through 180 per $\mu \mathrm{m}$. (Bottom) The beam exiting the WP's intensity is shown in the bottom plot. If the incident state is polarized at angle $\theta$, the bottom curve is shifted in phase by $\theta$, providing the mechanism to measure AoLP. If the DoLP is less than 1 , the amplitude of the modulation is reduced

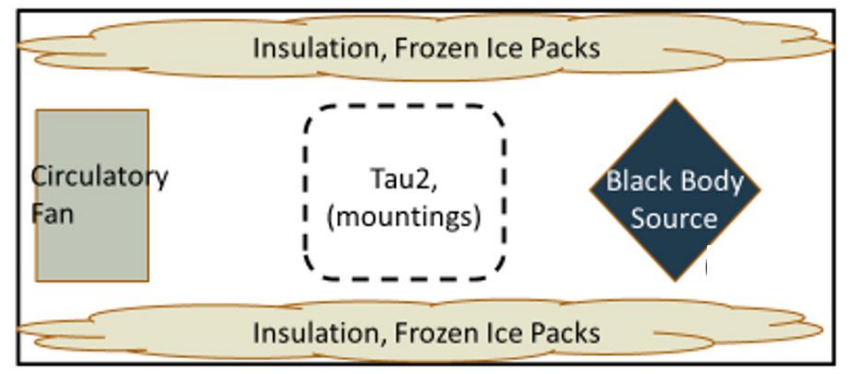

Figure 2. Layout of environmental chamber design. Water and dry ice are used to modulate the temperature inside of the chamber. Chemical desiccant is also included to prevent excess humidity

\subsection{System Characterization and Evaluation}

Polarization effects and operation of the system are modeled in Polaris-M, an advanced polarization software developed for polarization modeling by Airy Optics. ${ }^{9}$ The retrieval of the AoLP and DoLP values from the intensity spectrum is dependent on the precise modeling of transmission losses throughout the system. Thus, wavelength dependent diattenuation must be modeled and measured for each element. As the most significant transmission loss is introduced by the diffraction grating, special diattenuation considerations are made for the grating using Polaris' built in Rigorous Coupled Wave Analysis (RCWA) algorithms.

\subsection{Microbolometer Calibration}

The performance of the IRCSP is enabled by recent advances in calibration for long term stability in uncooled microbolometer arrays. ${ }^{10}$ Successful pixel by pixel calibration and well studied radiometric responses are essential to differentiate the polarization signal from camera performance artifacts. Successful long term calibration of our camera, FLIR's Tau2, has been demonstrated by Nugent and Shaw by recording long term response measurements over slowly varying focal plane temperatures. ${ }^{10}$ To perform these calibrations, an environmental chamber (Figure 2) has been constructed out of a commercially available ice chest, a circulatory fan, desiccant, and temperature sources. 


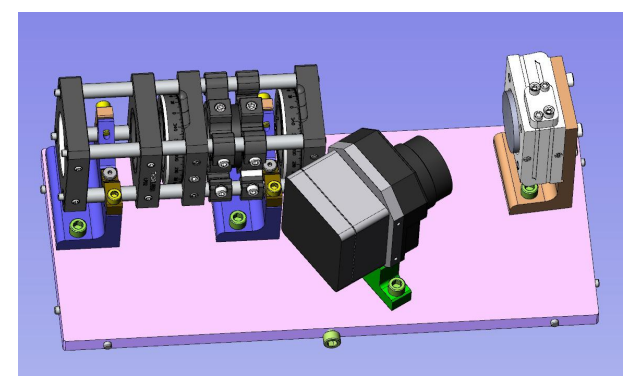

Figure 3. Table-top mechanical layout for ICRSP

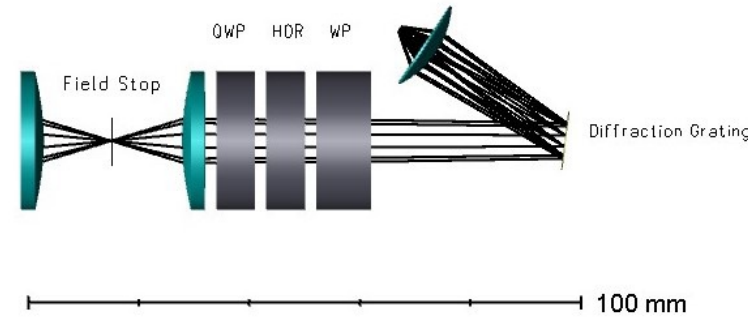

Figure 4. IRCSP optical subassembly

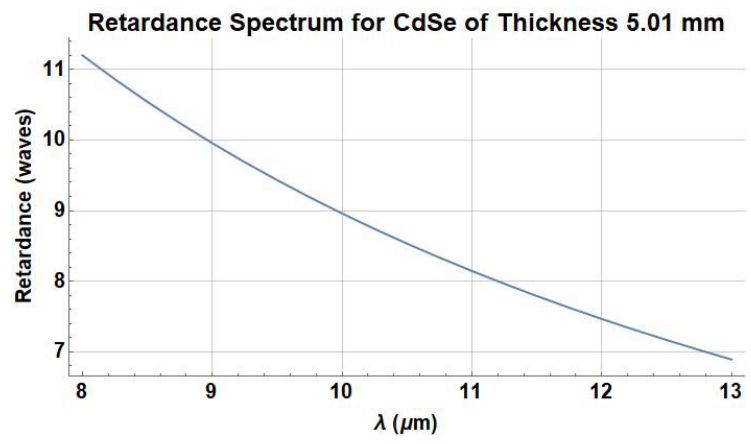

Figure 5. Retardance spectrum for HOR calculated in Polaris-M. The retardance difference $\delta_{8.5-12.5}=4$ waves as desired

\section{OPTICAL DESIGN}

\subsection{Polarization Modulation}

CdSe was selected as the birefringent material for the HOR and WP due to its thermal stability and well documented performance in the LWIR. ${ }^{11}$ An off the shelf cadmium thiogallate zero order wave-plate from Edmund Optics was purchased as the QWR. The HOR was designed to produce 4 waves of variation in retardance across 8.5-12.5 microns, approximately 7-11 waves of retardance. Using Polaris-M, the required thickness was calculated to be approximately $5 \mathrm{~mm}$. The retardance spectrum can be seen in Figure 5 . The crystal was manufactured and AR coated by Gooch and Housego in Ohio.

In the preliminary design, a linear polarizer was used in place of the WP to modulate the intensity. Once the Mueller matrix data reduction was modeled using atmospheric and system transmission data it became apparent that the ozone absorption band between 9.5 and 10.5 microns would produce a significant artifact to the order of 1 period, reducing the accuracy of measurements from 9.5-10.5 microns. Since the ozone band extends over one period of modulation (Figure 6), both $0^{\circ}$ and $90^{\circ}$ channels must be preserved to correct for the nature of the source spectrum. Since a Wollaston prism enables the imaging of both channels onto the same detector, it replaced the linear polarizer in the optical design.

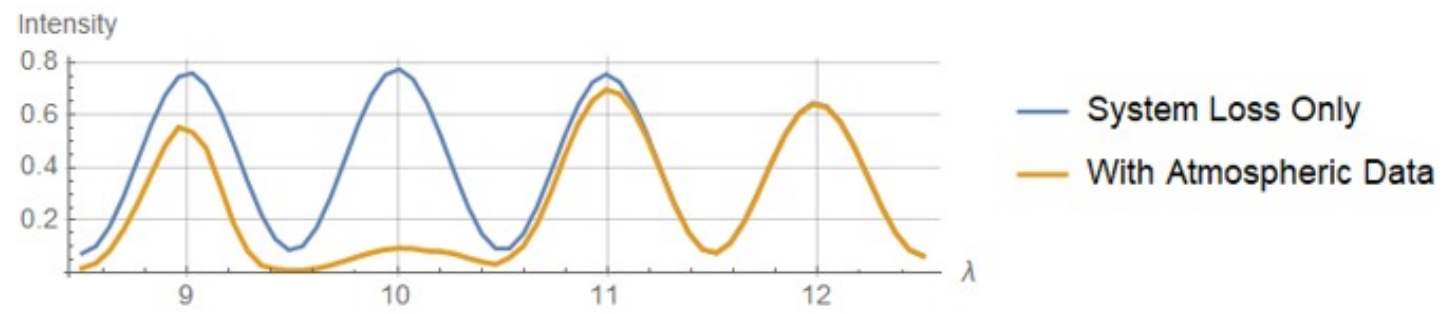

Figure 6. The intensity modulation at the detector for the p-polarization channel for AoLP $=45$ degrees and DoLP $=0.8$. The blue signal represents the produced intensity only considering transmission effects, and the orange signal is modeled using predictive atmospheric absorption losses. Without both polarization channels, it is ambiguous if the amplitude at 10 microns corresponds to a lower DoLP or absorption at that wavelength. 


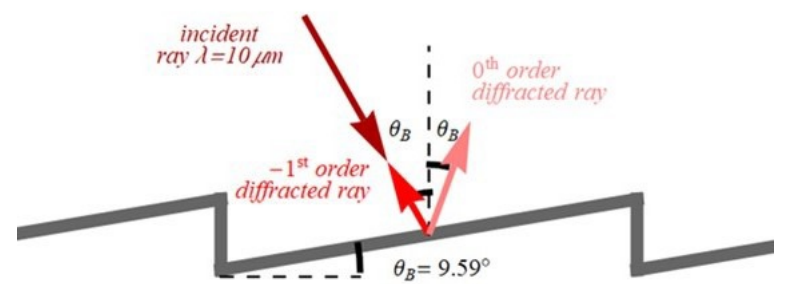

Figure 7. Blazed diffraction grating profile, grating period of $30 \mu \mathrm{m}$

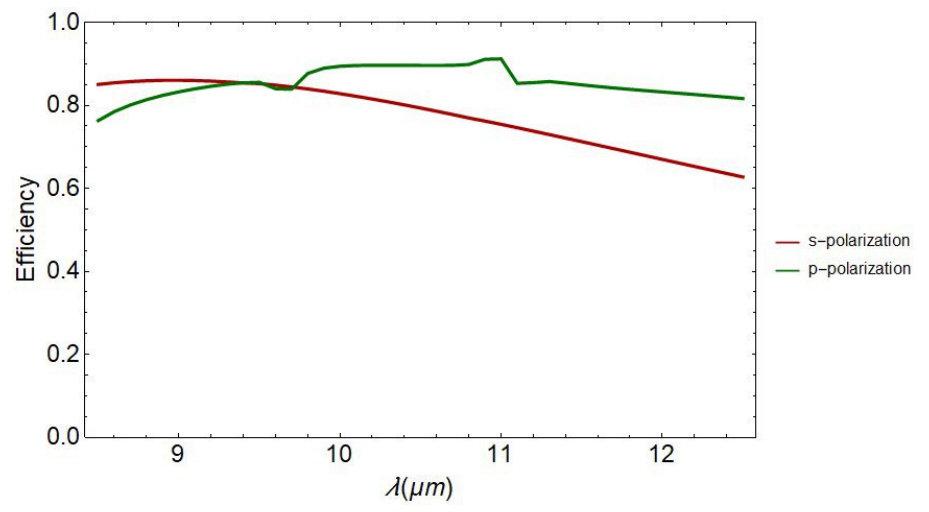

Figure 8. Grating efficiency for copper grating at $10.6 \mu m$ calculated using RCWA in Polaris-M

The successful fabrication and implementation of a CdSe WP has been demonstrated by the CanariCam polarimeter, which reported $>80 \%$ transmission across the 8.5-12.5 micron range. ${ }^{12}$ Polaris-M modeling and optimization of a CdSe Wollaston prism prescribe a desired prism angle of $\Omega=10.1^{\circ}$ to produce 30 pixels of separation between polarization channels.

\subsection{Diffraction Grating}

The grating spacing was designed to produce the desired dispersion on the detector given the camera's effective focal length and the pixel pitch. Using the grating equation, a grating period of 30 microns was calculated. A grating blaze angle was chosen to optimize the efficiency at 10.6 microns, resulting in the desired grating profile seen in Figure 7. The grating profile was then analyzed in Polaris-M for several materials and acceptable efficiency was verified. A copper substrate was chosen both for its high efficiency performance (Figure 8) and for ease of manufacturing. Since this custom grating specification is not a commercially available product, it was manufactured using diamond turning at the University of Arizona Precision Freeform Optics Design, Fabrication, and Testing Facility.

\section{CONCLUSION}

This paper describes a compact LWIR channeled spectro-polarimeter concept with the ability to resolve spectral and polarimetric data across the 8.5-12.5 micron band. The polarimetric measurements at these wavelengths will provide much needed measurements of bulk ice particle shape in clouds. ${ }^{3}$ As with any new instrument concept, the construction of the ICRSP is not without risk. At this stage in the SWIRP project, the mechanical layout and optical design for the IRCSP has been fully specified. However, further adjustments to the design will likely be needed to control thermal noise and prevent ghost images. For the polarization modulation, CdSe has been chosen as the birefringent material for the high order retarder and Wollaston prism, and the needed thicknesses and tolerances have been calculated. While the CdSe HOR is well studied, the relatively small difference between the extraordinary and ordinary refractive indices of the CdSe prevent a large dispersion angle to be achieved at the output of the Wollaston prism. Any change in the optical design may require an output dispersion greater than what is achievable with CdSe. Further study into alternate birefringent materials, such as Tellurium, is needed to mitigate the limitations of a CdSe Wollaston prism. A custom diffraction grating has 
been manufactured to meet the design specifications, but further efficiency studies must be preformed to ensure that laboratory measurements of the grating produce acceptable efficiencies. In addition, the final calibrated performance of the microbolometer may limit the resolving power of the polarimeter. Calibration studies of the camera, diffraction grating efficiency measurements, and stray light analysis will instruct modifications to the current design as the project moves into assembly and testing. The prototype ICRSP in on schedule to be fully assembled and begin preliminary performance evaluations in 2018.

\section{ACKNOWLEDGMENTS}

This work was supported by the NASA Goddard Space Flight Center and funded by NASA ESTO. The authors thank Rongguang Liang and the Precision Freeform Optics Design, Fabrication and Testing Facility at the University of Arizona for the fabrication of the custom diffraction grating. In addition, the authors thank Chang Jin Oh and Marco Favela of the University of Arizona Optical-Mechanical Design team for assistance in the system mechanical design. The authors also thank Adriana Stohn for her work developing the environmental chamber.

\section{REFERENCES}

[1] Buehler, S. A., Defer, E., Evans, F., Eliasson, S., Mendrok, J., Eriksson, P., Lee, C., Jiménez, C., Prigent, C., Crewell, S., Kasai, Y., Bennartz, R., and Gasiewski, A. J., "Observing ice clouds in the submillimeter spectral range: the cloudice mission proposal for esa's earth explorer 8," Atmospheric Measurement Techniques 5(7), 1529-1549 (2012).

[2] Council, N. R., [Earth Science and Applications from Space: National Imperatives for the Next Decade and Beyond], The National Academies Press, Washington, DC (2007).

[3] NASA, [Responding to the Challenge of Climate and Environmental Change: NASAs Plan for a ClimateCentric Architecture for Earth Observations and Applications from Space] (2010).

[4] Julia Craven-Jones, Brandyn M. Way, J. H. M. W. K. J. A. M., "Thermally stable imaging channeled spectropolarimetry," (2013).

[5] Frank J. Iannarilli, Joseph A. Shaw, S. H. J. H. E. S., "Snapshot lwir hyperspectral polarimetric imager for ocean surface sensing," (2000).

[6] Frank J. Iannarilli, Stephen H. Jones, H. E. S. P. L. K., "Polarimetric-spectral intensity modulation (p-sim): enabling simultaneous hyperspectral and polarimetric imaging," (1999).

[7] Herman E. Scott, Stephen H. Jones, F. J. I., "Imaging infrared polarimetry: initial results and potential in detection of scatterable mines and surface disturbances," (1999).

[8] Diner, D. J., Davis, A., Hancock, B., Gutt, G., Chipman, R. A., and Cairns, B., "Dual-photoelasticmodulator-based polarimetric imaging concept for aerosol remote sensing," Appl. Opt. 46, 8428-8445 (Dec 2007).

[9] Russell A. Chipman, W. S. T. L., "The polaris-m ray tracing program," (2015).

[10] Paul W. Nugent, J. A. S., "Calibration of uncooled lwir microbolometer imagers to enable long-term field deployment," (2014).

[11] Chenault, D. B. and Chipman, R. A., "Infrared birefringence spectra for cadmium sulfide and cadmium selenide," Appl. Opt. 32, 4223-4227 (Aug 1993).

[12] Christopher C. Packham, Rachel E. Mason, G. D. B., "Birefringence and transmission of an antireflectioncoated sulfur-free cadmium selenide wollaston prism at 30 k," Optical Engineering 47, $47-47-7$ (2008). 